\title{
Coordenação motora de idosas
}

\section{Motor coordination of elderly women}

\author{
Juliana Izabel Katzerl; Danielle Ledur Antes2; Sara Teresinha Corazza \\ ${ }^{1}$ Mestre em Educação Física - UFPel. Pelotas, RS - Brasil. \\ ${ }^{2}$ Mestre em Educação Física - UFSC. Florianópolis, SC - Brasil. \\ ${ }^{3}$ Professora Adjunta do Departamento de Métodos e Técnicas Desportivas do Centro de \\ Educação Física e Desportos - UFSM. Santa Maria, RS - Brasil. \\ Endereço para correspondência \\ Juliana Izabel Katzer \\ R. Dr. Liberato Salzano Vieira, 205. Apto. 3, Bairro Camobi \\ 97105-404 - Santa Maria - RS [Brasil] \\ julikatzer@gmail.com
}

\begin{abstract}
Resumo
Introdução: $\mathrm{O}$ envelhecimento pode causar alterações físicas, motoras e cognitivas. Para minimizar esses efeitos, o idoso deve manter-se ativo fisicamente e assim melhorar os elementos motores fundamentais, como a coordenação motora, para garantir boas condições de saúde. Objetivo: Avaliar a coordenação motora de idosas praticantes e não praticantes de exercícios físicos. Métodos: Participaram 50 idosas, com idade média de 65,91 $\pm 2,05$ anos, sendo 25 ativas (G1); e 25, não ativas (G2). Para avaliar a coordenação motora, utilizou-se o teste de habilidades manuais de Andreotti e Okuma ${ }^{5}$. Resultados: Os tempos médios para executar a tarefa foram $7,72 \pm 1,79$ segundos (G1) e 9,28 $\pm 2,51$ segundos (G2). O teste de coordenação motora apresentou diferença significante $(Z=-2,354, p<0,02)$ entre os grupos. Conclusão: As idosas fisicamente ativas (G1) apresentaram desempenho superior na execução da tarefa, o que evidencia os benefícios da prática regular de exercícios físicos para a manutenção dos elementos de uma estrutura motora básica.
\end{abstract}

Descritores: Atividade motora; Desempenho psicomotor; Destreza motora; Envelhecimento; Exercício físico.

\begin{abstract}
Introduction: Aging can cause physical, motor and cognitive changes. To minimize these effects, the elderly should remain physically active in order to improve fundamental motor skills, such as motor coordination, to ensure a good state of health. Objective: To evaluate the motor coordination of elderly women who practice physical activity and those who do not. Methods: A total of 50 elderly women with a mean age of $65.91 \pm 2.05$ years participated in this study, of which 25 practiced physical activity (G1) and 25 did not (G2). In order to evaluate motor coordination, the manual skills test of Andreotti and Okuma ${ }^{5}$ was used. Results: The mean times for performing the task were $7.72 \pm 1.79$ seconds (G1) and $9.28 \pm 2.51$ seconds (G2). The motor coordination test showed a significant difference $(Z=-2,354, p<0.02)$ between groups. Conclusion: The physically active elderly women (G1) showed superior performance in executing the required task, demonstrating the benefits of regularly practicing physical activity to maintain basic motor skill.
\end{abstract}

Key words: Aging; Exercise, physical; Motor activity; Motor skills; Psychomotor performance. 


\section{Introdução}

O aumento da proporção de idosos na população brasileira traz à tona a discussão de propostas para contribuir com um envelhecimento saudável e com melhor qualidade de vida. De acordo com a Pesquisa Nacional de Amostra e Domicílio (PNAD), realizada no Brasil em 2007, $10,5 \%$ da população brasileira apresentava idade igual ou superior a 60 anos $^{1}$.

Um dos grandes desafios nos últimos anos é garantir à população idosa qualidade de vida, com o intuito de promover melhores condições de saúde para essas pessoas.

Esse desafio tem despertado o interesse em entender, nos vários domínios, as alterações físicas - tais como perda de massa muscular e mudanças da estrutura esquelética; alterações motoras - decréscimos de habilidades, como, por exemplo, força, equilíbrio, flexibilidade - e alterações cognitivas - perda de memória e da capacidade de raciocínio rápido entre outras, relacionadas com o aumento da idade, e os processos que as desencadeiam. Dessa forma, percebese que o envelhecimento se traduz em aumento das incapacidades e, portanto, cada vez mais, busca-se encontrar "soluções" para minimizar as perdas funcionais ocasionadas pelo envelhecimento $^{2}$. Entre as principais formas de evitar e/ou minimizar os declínios físicos, psicológicos e sociais que frequentemente acompanham os idosos, tem-se a atividade física, a qual vem mostrando amplas evidências de associação com melhoras significativas nas condições de saúde como o controle do estresse, obesidade, diabetes, doenças coronarianas e, principalmente, na aptidão funcional ${ }^{3,4}$.

A atividade física tanto melhora a capacidade muscular como pode melhorar a resistência, o equilíbrio, a mobilidade articular, a agilidade, a velocidade da caminhada e a coordenação geral 5 . Outros estudos, buscando amenizar as alterações do envelhecimento, utilizam-se de atividade física com enfoques nas habilidades básicas, como equilíbrio, força, flexibilidade, tempo de reação e coordenação, as quais são elementos fundamentais para a realização das atividades da vida diária ${ }^{6}$.

Dentre essas, destaca-se a coordenação motora, importante elemento na realização de tarefas, como, dirigir um carro, caminhar, dançar, usar talheres, discar um número telefônico, pegar moedas, escovar os dentes, pentear-se.

Alguns estudos mostram a melhoria da coordenação a partir da participação em exercícios físicos, como em treinos de movimentos de força de punho e mão ${ }^{7}$, de hidroginástica ${ }^{8}$ e de Tai Chi Chuan'. Em vários estudos 7, 8, 9, 10, 11, 12, 13, verificaram-se - a partir da aplicação do teste de coordenação proposto pela American Alliance for Health, Physical Education, Recreation and Dance (AAHPERD) - melhoras nos níveis de coordenação em idosos submetidos a um programa de atividade física generalizada de intensidade moderada; porém, estudos comparando a coordenação motora de idosas praticantes e não praticantes de atividades físicas regulares são menos frequentes.

Nesse sentido, o propósito neste estudo foi testar a hipótese de que idosas que praticam atividades físicas regulares possuem uma melhor coordenação motora, quando comparadas com idosas não praticantes dessas atividades.

\section{Materiais e métodos}

\section{População e amostra}

A amostra foi intencionalmente selecionada pela idade, ou seja, a participante deveria ter 60 anos ou mais. O grupo de idosas praticantes de atividades físicas regulares (G1) foi constituído por mulheres que participavam de um programa de atividades de hidroginástica para a terceira idade há no mínimo três meses, com frequência mínima de duas vezes na semana. Já as idosas não praticantes de atividades físicas regulares (G2), foram assim designadas após responderem uma anamnese na qual relataram não praticar nenhum tipo de atividade física com frequência ou intensidade controlada, mencionaram realizar apenas atividades domésticas. 
Todas as participantes foram devidamente esclarecidas sobre a investigação e assinaram o Termo de Consentimento Livre e Esclarecido, seguindo as exigências para pesquisas que envolvem seres humanos, de acordo com a Resolução 196 de 1996 do Conselho Nacional de Saúde, após aprovação do estudo pelo Comitê de Ética em Pesquisa, composto por professores e membros da comunidade vinculados à Universidade Federal de Santa Maria (UFSM), sob o número de protocolo 076024300006 .

\section{Procedimentos do estudo}

Para identificar e separar os grupos, foi realizada uma anamnese e investigadas as atividades praticadas pelas idosas. A coordenação motora foi avaliada por meio do teste de habilidades manuais ${ }^{5}$.

O teste foi aplicado no Laboratório de Aprendizagem Motora do Centro de Educação Física e Desportos da Universidade Federal de Santa Maria. Ressalta-se que as coletas realizaram-se individualmente, sem interferências, em lugar calmo e sem ruídos.

\section{Anólise estatística}

A análise estatística foi realizada por meio do Software Statistical Package for the Social Sciences, versão 13.0, e por meio do teste U de Mann Whitney para verificar a diferença entre os grupos, considerando nível de significância de 5\%.

\section{Resultados}

Foram selecionadas 50 idosas, com média de idade de 65,91 $\pm 2,05$ anos, dividas em grupos, sendo 25 praticantes de atividades físicas regulares (G1) e 25 não praticantes de atividades físicas regulares (G2).

Na Tabela 1, apresentam-se os resultados obtidos no teste de coordenação motora por ambos os grupos (G1 e G2). Foi verificada uma diferença estatisticamente significante entre os grupos $(Z=-2,354, p<0,05)$, o que deixa claro que as idosas do G1 obtiveram um desempenho superior em relação às do $\mathrm{G} 2$, fato explicado pela prática regular da atividade física.

Tabela l: Análise descritiva (tempo em segundos). Gl - idosas praticantes de atividades físicas regulares; G2 - idosas não praticantes de atividades físicas regulares

\begin{tabular}{cccccc}
\hline Grupos & Mínimo & Máximo & Média & $\begin{array}{c}\text { Desvio- } \\
\text { padrão }\end{array}$ & p valor \\
\hline $\mathrm{G} 1$ & $6,00 \mathrm{~s}$ & $9,00 \mathrm{~s}$ & $7,72 \mathrm{~s}$ & 0,92 & \\
$\mathrm{G} 2$ & $6,01 \mathrm{~s}$ & $14,03 \mathrm{~s}$ & $9,28 \mathrm{~s}$ & 2,52 & $0,019^{*}$ \\
\hline
\end{tabular}

*diferença significante $(p<0,05)$.

A Figura 1 apresenta o desempenho das idosas no teste. Nota-se que as componentes do G1 mantiveram um desempenho semelhante entre si, enquanto que as do G2 mostraram uma variação entre elas, que é representada pelo alto desvio-padrão visto na Tabela 1.



Figura 1: Valores obtidos pelas idosas no teste de habilidades manuais

\section{Discussão}

Os resultados deste estudo apontam para os benefícios da atividade física, já que as idosas praticantes de atividades físicas regulares tiveram um desempenho superior às não praticantes dessas atividades na realização da tarefa proposta. Fato que corrobora outros autores que afirmam que a pessoa idosa sedentária tem como característica uma diminuição do rendimento 
motor, pois ocorre evidente regressão nas capacidades de velocidade, equilíbrio, agilidade, força, resistência ${ }^{3}$ e também nos movimentos mais precisos das mãos e dos pés, por ocorrer gradativamente um atrofiamento desses segmentos.

Em um estudo com idosas praticantes de exercícios em meio líquido ${ }^{14}$, em que se analisou o nível de propriocepção e coordenação motora fina em um grupo de estudos composto por 56 mulheres, entre 59 e 69 anos de idade, tiveram um tempo médio de oito segundos para realizar a mesma tarefa utilizada nesta pesquisa. Tais resultados mostram-se semelhantes aos encontrados nesta investigação.

Após acompanhar por 12 anos dois grupos de mulheres idosas ${ }^{15}$ (um grupo que praticava atividade física e outro que apenas executava atividades de vida diária), com média de 65,6 anos, encontraram-se resultados melhores para todas as capacidades investigadas, incluindo a coordenação, no grupo de idosas ativas. Enquanto que o grupo de voluntárias que executavam apenas atividades diárias apresentou declínio nos níveis de coordenação. Resultados esses que reforçam os achados do estudo aqui apresentado, indicando que idosas praticantes de atividades físicas regulares têm coordenação melhor. Confirma-se assim, que a atividade física traz melhoras nos níveis de coordenação motora em sujeitos idosos. Analisando os efeitos de um programa regular e sistematizado de dança sobre os componentes da capacidade funcional (flexibilidade, agilidade e equilíbrio dinâmico, resistência de força, coordenação motora e resistência aeróbia) em mulheres acima de 50 anos $^{16}$, verificou-se que a coordenação motora (óculo-manual) apresentou melhoras significativas nas idosas após quatro meses de prática de dança. Demonstrando novamente, que a atividade física traz melhoras nos níveis de coordenação motora em sujeitos idosos.

Quando analisada a eficiência da prática de atividade física ${ }^{17}$ por meio do efeito de 19 meses de ginástica recreativa na coordenação motora fina de 70 idosos, com idade entre 60 e 90 anos, antes e após a realização das aulas, os resultados também mostraram melhora significante para a coordenação motora fina. Isso foi observado mesmo quando alguns sujeitos apresentaram comprometimento nos dedos das mãos e dos pés, dificultando a flexão e extensão dos membros.

Em outro estudo ${ }^{18}$, que objetivou avaliar o efeito de um treinamento de ginástica rítmica na destreza e velocidade de membros superiores em idosas, tendo como amostra um grupo formado por 30 mulheres, divididas em grupo experimental e controle, fisicamente ativas, na faixa etária média de 69 anos, tendo como tratamento experimental 30 minutos semanais de treinamento, durante oito semanas consecutivas. Os resultados mostraram uma evolução significativa na velocidade e destreza de membros superiores após dois meses de treinamento a favor do grupo experimental.

Para comprovar os efeitos da atividade física na coordenação olho/mão de idosos em que foram avaliados 42 sujeitos, sendo 22 participantes de um programa de atividade física, e 20, do grupo controle. O grupo praticante apresentou melhores resultados para o teste de coordenação olho/mão, quando comparado ao controle. Isso mostra que idosos que mantêm uma prática regular de atividade física tendem a apresentar melhoras significativas na coordenação, o que pode ser fundamental para uma vida longa saudável e fisicamente independente ${ }^{9}$.

Diante desses achados, observa-se que a atividade física regular proporciona melhora da aptidão funcional de idosos, principalmente na coordenação motora, objeto de estudo desta pesquisa. Assim, indivíduos que mantêm uma atividade física regular tendem a minimizar os efeitos do envelhecimento nas capacidades motoras e a melhorar a qualidade de vida. Isso é observado em estudos em que a pessoa idosa sedentária tem como característica uma diminuição do rendimento motor pela evidente regressão nas capacidades de velocidade, equilíbrio, agilidade, força, resistência e, principalmente, de movimentos mais precisos das mãos e dos pés, por ocorrer gradativamente um atrofiamento desses segmentos ${ }^{19,20}$. 


\section{Conclusão}

Os resultados deste estudo reforçam a ideia de que idosos fisicamente ativos, além de manterem e melhorarem a qualidade de vida, tornam-se independentes para as atividades de vida diária, tendo condições físicas e cognitivas para continuar desenvolvendo numerosas tarefas e papéis diversos na sociedade.

Os resultados comprovam a hipótese do estudo de que a prática regular de atividade física, durante o processo de envelhecimento, traz melhoras nos níveis da coordenação motora das idosas. Esse fato ressalta a importância de avaliar as modificações que ocorrem em razão dessa prática e assim, com esses dados, promover uma melhora na qualidade dos programas de exercícios físicos desenvolvidos para essa população.

\section{Referências}

1. Instituto Brasileiro de Geografia e Estatística. Projeção da População do Brasil: população brasileira envelhece em ritmo acelerado. [acesso em 2010 maio 18]. Disponível em: <http://www.ibge.gov. br/home/default.php $>$.

2. Matsudo SM, Matsudo VKR, Barros Neto TL de. Impacto do envelhecimento nas variáveis antropométricas, neuromotoras e metabólicas da aptidão física. Rev Bras Ciênc Mov. 2000 set;8(4):21-32.

3. Chodzko-Zajko WJ, Proctor DN, Fiatarone Singh MA, Minson CT, Nigg CR, Salem GJ, et al. American College of Sports Medicine position stand. Exercise and physical activity for older adults. Med Sci Sports Exerc. 2009 Jul;41(7):1510-30.

4. Gobbi S. Atividade física para pessoas idosas e recomendações da Organização Mundial de Saúde de 1996. Rev Bras Ativ Fís e Saúde. 1997;2(2):41-9.

5. Andreotti RA, Okuma SS. Validação de uma bateria de testes de atividades de vida diária para idosos fisicamente independentes. Rev Paul Educ Fís. 1999;13(1):46-66.

6. Santos S, Dantas L, Oliveira JA de. Desenvolvimento motor de crianças, de idosos e de pessoas com transtornos da coordenação. Rev Paul Educ Fís. 2004;8:33-44.
7. Contreras-Vidal JL, Bo J, Boudreau JP, Clark JE. Development of visuomotor representations for hand movement in young children. Exp Brain Res. 2005;162:155-64.

8. Antes DL, Katzer JI, Corazza ST. Coordenação Motora Fina e Propriocepção de Idosas Praticantes de Hidroginástica. Rev Bras Cienc Env Hum. 2008 jul-dez;5(2):24-32.

9. Pei Y, Chou S, Lin P, Lin Y, Hsu T, Wong A. Eye-hand coordination of elderly people who practice Tai Chi Chuan. J Formos Med Assoc. 2009;107(2):103-10.

10. Polastri PF, Silva VM, Villar R, Zago AS, Gobbi S. Alterações nos níveis de coordenação de pessoas da terceira idade através de um programa de atividade física generalizada. Rev Motriz. 1999;5(1):115.

11. Zago AS, Gobbi S. Valores normativos da aptidão funcional de mulheres de 60 a 70 anos. Rev Bras Cienc Mov. 2003;11(2):77-66.

12. Benedetti TRB, Gobbi S, Mazo GZ, Lopes MA, Gobbi L, Ferreira L, Hoefelmann CP. Valores normativos de aptidão funcional em mulheres de 70 a 79 anos. Rev Bras Cine Des Hum. 2007;9(1):28-36.

13. Ferreira L, Barbosa TD, Gobbi S, Arantes LM. Capacidade funcional em mulheres jovens e idosas: projeções para uma adequada prescrição de exercícios físicos. Rev Educ Fís. 2008;19(3):403-12.

14. Antes DL, Corazza ST. Nível de propriocepção e coordenação motora fina de idosas praticantes de hidroginástica. Fiep Bulletin. Special Edition. 2007;77:009.

15. Pauli JR, Souza LS, Zago AS, Gobbi S. Influência de 12 anos de prática de atividade física regular em programa supervisionado para idosos. Rev Bras Cine Des Hum. 2009;11(3):255-60.

16. Sebastião E, Hamanaka AYY, Gobbi LTB, Gobbi S. Efeitos da prática regular de dança na capacidade funcional de mulheres acima de 50 anos. Rev Educ Fis. 2008;19(2):205-14.

17. Monte ASI, Lages SMRN, Rodrigues MAS, Knackfuss MI. Ginástica recreativa: um paradigma para a coordenação motora do idoso. Fit \& Performance J. 2006;5(4):232-5.

18. Reis RGM, Santos FCP. Efeito de um treinamento com o aparelho arco da ginástica rítmica na destreza de mãos e dedos e velocidade de mãos e braços em idosas. Movimentum - Rev Dig Educ Fís. 2007 fev-jul;2(1).

19. Vale RGS, Torres JB, Martinho KO, Lopes RB, Novaes JS, Dantas EHM. Efeitos do treinamento de força na flexibilidade de mulheres idosas. Fit \& Performance J. 2004;3(5):266-71.

20. Braz ALO, Kroeff MS. A contribuição do treinamento resistido na promoção da saúde e qualidade de vida na terceira idade. FIEP Bulletin. 2006;76:98. 